\title{
XMM-Newton RGS spectra of four Seyfert 1 galaxies
}

\author{
M. V. Cardaci, ${ }^{1,2}$ M. Santos-Lleó ${ }^{2}$ and A. I. Díaz ${ }^{1}$ \\ ${ }^{1}$ Universidad Autónoma de Madrid, Cantoblanco, 28049-Madrid, Spain \\ ${ }^{2}$ XMM-Newton Science Operation Centre, ESAC, ESA, PO Box 50727, 28080 Madrid, Spain \\ email: Monica.Cardaci@esa.int
}

\begin{abstract}
Using the spectral fitting technique we have studied the components contributing to the soft X-ray spectra of four AGN. The selected objects are the Seyfert 1 galaxies: HE 11431810, Mkn 110, CTS A08.12 and ESO 359-G19. The high-resolution X-ray spectra analysed were taken with the Reflection Grating Spectrometers on board the XMM-Newton satellite. In contrast to the results for other well-studied Seyfert 1 galaxies, we have found that the spectra of the four galaxies lack of significant absorption features. Hence, there are no signs of highcolumn-density partially-ionised absorbing material in the vicinity of the active nucleus in these galaxies.
\end{abstract}

Keywords. galaxies: Seyfert - galaxies: individual (HE 1143-1810, CTS A08.12, ESO 359-G19, Mkn 110) - X-rays: galaxies

We have performed a simultaneous fitting of the RGS1 and RGS2 spectra of each of the sources in Table 1. To fit the continuum we used an absorbed power law with column density fixed to the Galactic values. For three of the sources, the addition of an intrinsic neutral absorption component significantly improved the fit. Finally, we added some Gaussian emission lines. Table 2 lists the values of the best fitting parameters. Figure 1 shows the spectra and the best model for Mkn 110.

As it can be seen in Table 2 the power-law indexes found are in the range of 2.2 to 3.7. These values are similar to those found in other AGN showing a soft-X-ray excess. Therefore, the indexes in Table 2 are probably indicating the presence of soft excess emission. For Mkn 110, CTS A08.12 and ESO 359-G19 we have found signatures of an intrinsic cool absorbing material with column densities between 0.1 and $3.6 \times 10^{21} \mathrm{~cm}^{-2}$. We have found oxygen emission lines in three of the four objects. The large errors on the OvII triplet lines in HE 1143-1810 and Mkn 110 do not allow charaterisation of electronic temperatures and densities of the emitting medium as explained in Gabriel \& Jordan (1969) and Porquet \& Dubau (2000). There are no indications of the presence of warm absorbers in the line-of-sight towards any of these four active nuclei.

Table 1. Observation details. In the last column we have included the galactic column densities estimated for the positions of the objects.

\begin{tabular}{lcrrrrr}
\hline \multicolumn{1}{c}{ Name } & Obs. ID & Obs. date & exp. time & \multicolumn{2}{c}{$10^{-2}$ counts/s } & Gal $n_{H}$ \\
& & & (s) & \multicolumn{1}{c}{ RGS1 } & \multicolumn{1}{c}{ RGS2 } & $\left(\mathrm{cm}^{-2}\right)$ \\
\hline HE 1142-1810 & 0201130201 & 8 Jun 2004 & 31000 & $90.4 \pm 0.6$ & $98.7 \pm 0.6$ & $3.4 \times 10^{20}$ \\
Mkn 110 & 0201130501 & 15 Nov 2004 & 47000 & $81.3 \pm 0.4$ & $88.4 \pm 1.8$ & $1.42 \times 10^{20}$ \\
CTS A08.12 & 0201130301 & 30 Oct 2004 & 46000 & $8.1 \pm 0.3$ & $9.6 \pm 0.3$ & $4.07 \times 10^{20}$ \\
ESO 359-G19 & 0201130101 & 9 Mar 2004 & 24000 & $2.8 \pm 0.3$ & $3.4 \pm 0.3$ & $1.02 \times 10^{20}$ \\
\hline
\end{tabular}



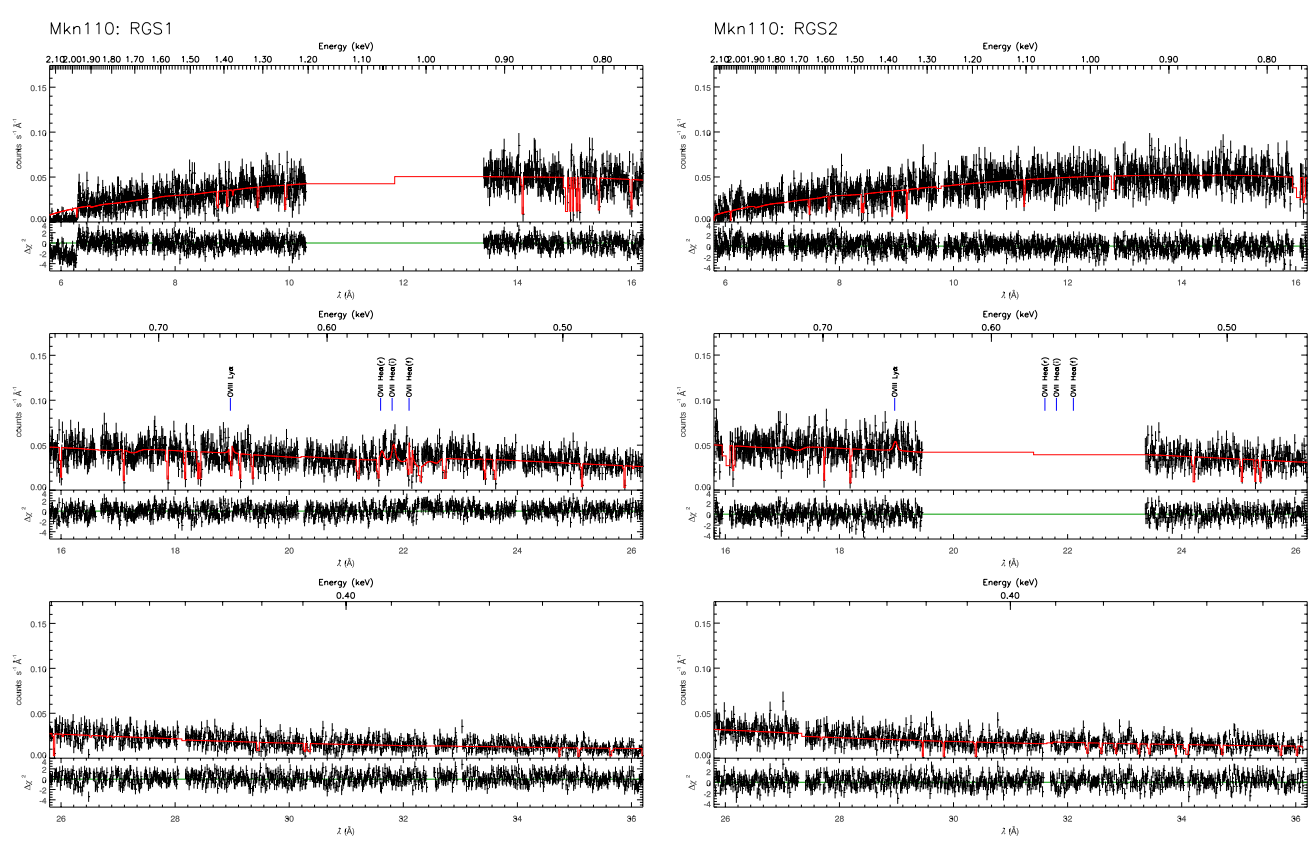

Figure 1. RGS spectra, plotted in rest frame, and the model fit (in red) for Mkn 110.

Table 2. Best fit parameters obtained for the spectra of the four active nuclei. The line fluxes are given in $10^{-14} \mathrm{erg} \mathrm{cm} \mathrm{cm}^{-2} \mathrm{~s}^{-1}$; power-law normalizations in $10^{-4}$ photons $\mathrm{keV}^{-1} \mathrm{~cm}^{-2} \mathrm{~s}^{-1}$.

\begin{tabular}{|c|c|c|c|c|c|}
\hline Component & Parameter & HE $1143-1810$ & Mkn 110 & CTS A08.12 & ESO 359-G19 \\
\hline Intrins. mat. & $n_{H}\left(\frac{10^{21}}{\mathrm{~cm}^{-2}}\right)$ & - & $0.11 \pm 0.03$ & $1.25 \pm 0.09$ & $3.6 \pm 0.9$ \\
\hline \multirow[t]{2}{*}{ Power law } & $\Gamma$ & $2.42 \pm 0.02$ & $2.24 \pm 0.04$ & $2.39 \pm 0.06$ & $3.7 \pm 0.6$ \\
\hline & norm & $120 \pm 1$ & $104 \pm 1$ & $22.3 \pm 0.5$ & $15_{-3}^{+4}$ \\
\hline \multirow[t]{2}{*}{ Ovir(r) line } & $E(\mathrm{keV})$ & $0.5717 \pm 0.0005$ & $0.5734 \pm 0.0003$ & - & - \\
\hline & flux & $6 \pm 3$ & $3_{-2}^{+3}$ & - & - \\
\hline \multirow[t]{2}{*}{ Ovir(i) line } & $E(\mathrm{keV})$ & 0.5663 & 0.5680 & - & - \\
\hline & flux & $3_{-3}^{+4}$ & $6 \pm 2$ & - & - \\
\hline \multirow[t]{2}{*}{ Ovir(f) line } & $E(\mathrm{keV})$ & 0.5587 & 0.5604 & - & - \\
\hline & flux & $9_{-6}^{+5}$ & $8_{-3}^{+2}$ & - & - \\
\hline \multirow[t]{2}{*}{ OviII Ly $\alpha$ line } & $E(\mathrm{keV})$ & - & $0.6530_{-0.0008}^{+0.0136}$ & $0.6531_{-0.0001}^{+0.0072}$ & - \\
\hline & flux & - & $4 \pm 2$ & $6 \pm 2$ & - \\
\hline Fit statistic & $\chi^{2} /$ dof & $5074.6 / 5219$ & $5667.6 / 5353$ & $5010.4 / 5190$ & $5142.1 / 5217$ \\
\hline Flux $0.5-2.0 \mathrm{keV}$ & $\left(\frac{\mathrm{erg}}{10^{12} \mathrm{~cm}^{2} \mathrm{~s}}\right)$ & $23.91 \pm 0.08$ & $21.36 \pm 0.05$ & $3.0 \pm 0.2$ & $1.1_{-0.5}^{+0.2}$ \\
\hline
\end{tabular}

\section{References}

Gabriel, A. H. \& Jordan, C. 1969, MNRAS, 145, 241

Porquet, D. \& Dubau, J. 2000, A\&A, 143, 495 